RETHINKING THE ROLE OF NAIRU

IN MONETARY POLICY: IMPLICATIONS

OF MODEL FORMULATION

AND UNCERTAINTY

Arturo Estrella

Frederic S. Mishkin

Working Paper 6518 


\section{RETHINKING THE ROLE OF NAIRU \\ IN MONETARY POLICY: IMPLICATIONS \\ OF MODEL FORMULATION \\ AND UNCERTAINTY}

Arturo Estrella

Frederic S. Mishkin

Working Paper 6518

http://www.nber.org/papers/w6518

\section{NATIONAL BUREAU OF ECONOMIC RESEARCH 1050 Massachusetts Avenue \\ Cambridge, MA 02138 \\ April 1998}

Prepared for the National Bureau of Economic Research conference on Monetary Policy Rules, Islamorada, Florida, January 15-17, 1998. We thank participants at the conference and at a seminar at the Federal Reserve Bank of New York for their helpful comments and Elizabeth Reynolds for excellent research assistance. The views expressed in this paper are those of the authors and do not necessarily represent those of the Federal Reserve Bank of New York, the Federal Reserve System, Columbia University or the National Bureau of Economic Research.

(C) 1998 by Arturo Estrella and Frederic S. Mishkin. All rights reserved. Short sections of text, not to exceed two paragraphs, may be quoted without explicit permission provided that full credit, including $(\mathcal{C}$ notice, is given to the source. 
Rethinking the Role of NAIRU in Monetary Policy:

Implications of Model Formulation and Uncertainty

Arturo Estrella and Frederic S. Mishkin

NBER Working Paper No. 6518

April 1998

JEL No. E5

ABSTRACT

In this paper we rethink the NAIRU concept and examine whether it might have a useful role in monetary policy. We argue that it can, but success depends critically on defining NAIRU as a short-run concept and distinguishing it from a long-run concept like the natural rate of unemployment. We examine what effect uncertainty has on the use of NAIRU in policy. Uncertainty about the level of NAIRU does not imply that monetary policy should react less to the NAIRU gap. However, uncertainty about the effect of the NAIRU gap on inflation does require adjustments to the policy reaction function. Also, as in Brainard (1967), uncertainty about the effect of the monetary policy instrument on the NAIRU gap reduces the magnitude of the policy response. We estimate a simple NAIRU gap model for the United States to obtain quantitative measures of uncertainty and to assess how these measures affect our view of the policy reaction function.

Arturo Estrella

Federal Reserve Bank of New York 33 Liberty Street

New York, NY 10045

arturo.estrella@ny.frb.org
Frederic S. Mishkin

Uris Hall 619

Graduate School of Business

Columbia University

New York, NY 10027

and NBER

fsm3@columbia.edu 


\section{Introduction}

Because the effects of monetary policy on the aggregate economy have long lags, monetary policy must necessarily be preemptive, that is, it must act well before inflation starts to rise. ${ }^{1}$ This, of course, is easier said than done. In order to act preemptively, monetary policymakers must have signals that help them forecast future changes in inflation. One such signal that has received substantial attention both in the academic literature and in the press is the gap between unemployment and NAIRU, the non-accelerating inflation rate of unemployment. ${ }^{2}$ In other words, NAIRU is the unemployment rate at which inflation is expected to neither increase or decrease.

The NAIRU concept has come under quite serious attack in recent years. In the early to mid 1990s, the common view in the economics profession was that NAIRU in the United States was around six percent. However, when the unemployment rate began to fall below six percent in 1995 and remained well below that level thereafter without any increase in inflation -- indeed inflation actually fell -- concern arose that the NAIRU concept might be seriously flawed. In addition, recent academic research has shown that there is great uncertainty in the estimates of NAIRU (e.g., Staiger, Stock and Watson (1997a,b)), suggesting that looking at the unemployment rate relative to NAIRU might not be a very helpful guide for monetary policy.

In this paper, we rethink the NAIRU concept and examine whether NAIRU might have a useful role in monetary policymaking. We argue that the answer is yes. However, the positive answer depends critically on redefining NAIRU very carefully and distinguishing it from a longrun concept like the natural rate of unemployment, something that is not typically done in the literature. Furthermore, as we will see, the view that the NAIRU concept implies that the 
monetary authorities should try to move the economy towards the NAIRU, thus to some extent treating it as a target, is both incorrect and misguided.

The first step in our analysis, in section 2, is to think about defining NAIRU in the context of setting monetary policy instruments. We adopt a definition that focuses on NAIRU as a reference point for monetary policy, and show that our definition of NAIRU is a short-run concept and is not the same as the natural rate of unemployment. Understanding that short-run NAIRU and the natural rate of unemployment differ is important, not only for the theoretical analysis to follow, but also because it suggests that the short-run NAIRU is likely to be highly variable, in contrast to the natural rate of unemployment. One immediate implication is that thinking of NAIRU as a level at which the unemployment rate should settle is not very useful for policy purposes.

Once we have defined the short-run NAIRU, we then go on to examine how it might be used in policymaking. We do this in several steps. First we look in section 3 at the certaintyequivalent case, when only inflation enters the policymakers' objective function and then when unemployment (or equivalently, output) as well as inflation are part of policymakers' objectives. Although the certainty-equivalent case is useful as a starting point for the analysis, we cannot stop here because there are several sources of uncertainty that have important implications for how monetary policy should be conducted. In addition to uncertainty about estimates of the actual value of NAIRU, there is uncertainty about the estimated parameters of the model, especially the parameters that measure the effect of the NAIRU gap on inflation and the impact of monetary policy instruments on the NAIRU gap. We examine in section 4 what effect these sources of uncertainty have on how the short-run NAIRU might be used in monetary 
policymaking, again under the pure price stability objective and then when unemployment as well as inflation enter the policymakers' objective function.

Our theoretical analysis shows that uncertainty about the level of the short-run NAIRU does not necessarily imply that monetary policy should react less to the NAIRU gap. However, uncertainty about the effect of the NAIRU gap on inflation does require an adjustment to the reference point for monetary tightening in terms of the level of unemployment and to the weight applied to the gap between actual and target inflation. Furthermore, as in Brainard (1967), uncertainty about the effect of the monetary policy instrument on the NAIRU gap reduces the magnitude of the policy response.

There is another sense in which uncertainty about the NAIRU may have an effect on policy. There may be uncertainty not just about the level of NAIRU or its effect, but about the way it is modeled: the exact form of the model specification may be unknown. Errors in model selection may result in excess uncertainty regarding both inflation forecasts and the parameters of the model. Thus, model selection has the potential to increase uncertainty about the effect of the NAIRU gap and to reduce the effectiveness of policy, and the magnitude of this problem may be more difficult to determine than that of simple parameter uncertainty. In section 5 , we focus on the losses associated with leaving out key information from the model.

Although our theoretical framework shows the qualitative effects of uncertainty on how monetary policy should be conducted, it cannot tell us whether these effects are economically important. To examine this question, we estimate in section 6 a simple NAIRU gap model for the United States to obtain quantitative measures of uncertainty and to assess how these measures affect our view of the optimal reaction of monetary policy to movements in unemployment 
relative to short-run NAIRU. Using an analogous model based on monthly data, we then examine how in practice the short-run NAIRU concept could be used in the actual conduct of monetary policy. The estimated models provide us with measures of short-run NAIRU that indicate that it is highly variable, suggesting that trying to drive the unemployment rate toward NAIRU, whether it is a short-run or a long-run concept, would be an inappropriate way to think about how monetary policy should be conducted. In particular, we use our analysis to evaluate whether the setting of monetary policy instruments in the face of rapidly falling unemployment rates in recent years makes sense.

\section{Defining Short-Run NAIRU: Why It Differs from the Natural Rate of Unemployment}

The concept of the natural rate of unemployment was first developed by Friedman (1968) and Phelps (1968) to argue that there would be no long-run tradeoff between unemployment and inflation. The natural rate of unemployment is defined as the level of unemployment to which the economy would converge in the long run in the absence of structural changes to the labor market. An implication of this definition is that expansionary monetary policy that leads to higher inflation would not be able to produce lower unemployment on average. Indeed, as mentioned in Friedman (1968), higher inflation might even have the opposite effect of raising unemployment in the long run because it would interfere with efficient functioning of labor markets. The concept of a natural rate of unemployment leads to the following characterization of an expectations-augmented Phillips curve:

$$
\pi_{t}=\pi_{t}^{e}+\beta(L)\left(u_{t}-\bar{u}_{t}\right)+\delta^{\prime} z_{t}+\epsilon_{t}
$$


where

$\pi_{t}=$ inflation rate from $t-1$ to $t$

$\pi_{t}^{e}=$ inflation rate expected at $t-1$

$u_{t}=$ unemployment rate at time $t$

$\bar{u}_{t}=$ natural rate of unemployment at time $t$, which could be a constant, but could shift with structural changes in the economy

$z_{t}=$ a vector of variables such as supply shocks, which have zero ex-ante expectation $\epsilon_{t}=$ an unspecified disturbance term.

In order to estimate this expectations-augmented Phillips curve, researchers typically assume that the expected inflation can be measured as a distributed lag on past inflation and other variables, and that the inflation rate is integrated of order one, so that $\Delta \pi_{t}$ is stationary. The resulting Phillips curve is then:

$$
\Delta \pi_{t}=\beta(L)\left(u_{t}-\bar{u}_{t}\right)+\gamma(L) \Delta \pi_{t-1}+\delta^{\prime} z_{t}+\epsilon_{t}
$$

The NAIRU (non-accelerating inflation rate of unemployment) concept was first developed in a paper by Modigliani and Papademos (1975) and is defined as the rate of unemployment at which there is no tendency for inflation to increase or decrease. In empirical work such as Staiger, Stock and Watson (1997a,b) and Gordon (1997), NAIRU is viewed as being equivalent to the natural rate of unemployment, $\bar{u}_{t}$, in equation (2) and is typically estimated by assuming that $\bar{u}_{t}$ is a constant, a random walk, or a linear transformation of some step function or spline. ${ }^{3}$ 
For policy purposes, equation (1) indicates that it is perfectly appropriate to think about the unemployment gap, $u_{t}-\bar{u}_{t}$, as one determinant of changes in the rate of inflation, recognizing that other factors, represented by the past history of inflation and the $z_{t}$ variables, also affect the inflation process. However, current unemployment is frequently compared with the estimated value of NAIRU, and the resulting NAIRU gap is taken to be an indicator of inflationary pressure. Under a strong form of this view, if policymakers wish to drive inflation down, they need to raise the unemployment level above NAIRU, whereas if inflation is at its desired level, monetary policy needs to keep unemployment from falling below NAIRU.

Policy discussions, therefore, frequently focus on the difference between the current level of unemployment and the NAIRU as estimated above, in other words, on the variable that enters the first term of equation (1) in a distributed lag. This implicit comparison has the advantage of simplicity: it focuses the discussion on a single indicator of inflationary pressure, the unemployment gap, that we know from the model should be zero in long run equilibrium. However, this advantage is overwhelmed by a number of serious problems associated with this procedure.

First, monetary policy does not generally focus only on long run equilibrium, so the gap as defined above may be of limited usefulness. Second, even if equation (1) is viewed as a shortrun forecasting equation, the dependent variable is contemporaneous monthly or quarterly inflation, which is quite unlikely to be the policy target in practice. Third, the current unemployment gap is only one of many explanatory variables in the equation, including several lags of the gap itself. Focusing on only one variable gives an incomplete picture. Fourth, the 
equation may not even represent the optimal forecast of inflation, since other potentially important variables may be omitted.

Finally, focusing on the unemployment gap may create the impression that the goal of policy is to drive unemployment towards the NAIRU as a target level. As equation (1) illustrates, the current unemployment gap, $u_{t}-\bar{u}_{t}$, is only one of many explanatory variables in the Phillips-curve equation. The presence of lags of $\Delta \pi$ in the equation suggests that inflation may decelerate because expected inflation is falling, even if the unemployment rate is below the natural rate of unemployment. Similarly, if there have been favorable supply shocks, inflation in the future may decelerate even though the unemployment rate is well below the natural rate. The presence of lags of the unemployment gap suggests complicated dynamics in which a current negative unemployment could also be associated with decelerating inflation. The presence of many other variables besides the current unemployment gap in the expectations-augmented Phillips-curve equation therefore implies that the unemployment rate at which there is no tendency for inflation to rise or fall over the policy horizon can be quite different from the natural rate of unemployment, $\bar{u}_{t}$. In other words, it can be quite misleading to focus on NAIRU, as an estimate in equation (1) of the natural rate of unemployment, because it is not clear that the introduction of policy shocks designed to drive unemployment towards this characterization of NAIRU will do anything to control inflation either in the short run or in the long run.

Therefore, we propose an alternative way of thinking about the NAIRU as a reference point for unemployment that reflects inflationary pressures over the short- or intermediate-run policy horizon. The key idea is that the reference point for unemployment at which inflation will neither increase nor decrease over the relevant policy horizon, which can be thought of as a 
short-run NAIRU, not only embodies $\bar{u}_{t}$, the natural rate of unemployment, but also the other variables that help predict inflation. In other words, we would like to express the change in inflation over the relevant policy horizon as a function of $u_{t}-n_{t}$, where $n_{t}$ is an appropriately constructed short-run NAIRU.

Thus, suppose that the policy horizon for inflation is from $j$ to $j+k$ months ahead and define

$$
\Delta \pi_{t}^{(j, k)}=(1200 / k) \log \left(p_{t+j+k} / p_{t+j}\right)-100 \log \left(p_{t} / p_{t-12}\right)
$$

as the difference between current annual inflation and inflation over the policy horizon, where $p_{t}$ is the price level in month $\mathrm{t}$. We then construct equation (2)

$$
\Delta \pi_{t}^{(j, k)}=\alpha+\beta(L) u_{t}+\gamma(L) \Delta \pi_{t}+\delta^{\prime} x_{t}+\epsilon_{t}
$$

which is similar to equation (1), save for the dependent variable and the inclusion of a vector $x$ that contains any predetermined variables that help predict inflation at the targeted horizon. ${ }^{4}$ In order to express the change in inflation as a function of the difference between unemployment and a short-run NAIRU, equation (2) can always be rewritten as

$$
\Delta \pi_{t}^{(j, k)}=\beta_{0}\left(u_{t}-n_{t}\right)+\epsilon_{t}
$$

with

$$
n_{t}=\text { short-run NAIRU }=-\left(\alpha+(\beta(L)-\beta(0)) u_{t}+\gamma(L) \Delta \pi_{t}+\delta^{\prime} x_{t}\right) / \beta(0)
$$


where all the predictive power of the equation has been subsumed in the short-run NAIRU $n_{t}$. This short-run NAIRU is not an estimate of the long-run equilibrium natural rate, but a reference rate that represents the level of current unemployment that would correspond to a forecast of no inflation change over the policy horizon. ${ }^{5}$ Another important point that immediately falls out of this equation is that since the short-run NAIRU is related to past lags of unemployment, inflation and any other variables that help forecast changes in inflation, the short-run NAIRU may undergo substantial fluctuations even if the natural rate of unemployment is a constant.

Equation (3) has several important advantages over equation (1). In contrast to the conventional equation, the dependent variable in equation (3) is the change in inflation over the target horizon. Second, the current NAIRU gap, $u_{t}-n_{t}$, is the only explanatory variable in the equation and it subsumes all the predictive power of the equation. Third, the equation provides an optimal forecast of targeted inflation, given current information.

The analysis of this paper will focus on equations (2) and (3) and on our corresponding definition of short-run NAIRU. For the purposes of theoretical analysis, we use a simplified version of these equations with a limited lag structure. We return to the more general specification, however, when we consider empirical estimates using monthly data in section 6 .

\section{The Role of the NAIRU in Policy-Making: the Certainty-Equivalence Case}

\subsection{Objective Function with Inflation Only}

For the theoretical analysis, we start with a simple joint model of unemployment and inflation that is isomorphic to the one employed by Svensson (1997) with an output gap. In addition to inflation $\pi$ and an unemployment gap $\tilde{u}$, the model contains an exogenous variable $x$ 
and a monetary policy control variable $r$. This model will be the basis for the next few sections of the paper. However, some specific assumptions will be adjusted in subsequent sections in order to address particular issues. Assume for the purposes of this section that the parameters of the model are known with certainty.

$$
\begin{aligned}
& \pi_{t}=\pi_{t-1}-a_{1} \tilde{u}_{t-1}+a_{3} x_{t-1}+\epsilon_{t} \\
& \tilde{u}_{t}=b_{1} \tilde{u}_{t-1}+b_{2} r_{t-1}+b_{3} x_{t-1}+\eta_{t} \\
& x_{t}=c_{3} x_{t-1}+v_{t}
\end{aligned}
$$

where $\tilde{u}_{t}=u_{t}-\bar{u}$ and $r_{t}=$ the monetary policy variable. Equation (5) is a dynamic Phillips curve in which both unemployment and $x$ are predictors of inflation one-period ahead, say a year. Equation (6) is an IS curve and equation (7) defines the dynamics of the exogenous variable, $x$. The equilibrium level of all the variables is zero. Note, therefore, that the policy variable $r$ might be more similar to a change in the interest rate rather than the level.

The reduced form expression for inflation two periods ahead based on current values of the variables is

$$
\pi_{t+2}=\pi_{t}-a_{1}\left(1+b_{1}\right) \tilde{u}_{t}-a_{1} b_{2} r_{t}+\left(a_{3}\left(1+c_{3}\right)-a_{1} b_{3}\right) x_{t}+\xi_{t+2}
$$

where 


$$
\xi_{t+2}=-a_{1} \eta_{t+1}+a_{3} v_{t+1}+\epsilon_{t+1}+\epsilon_{t+2}
$$

Assume now that the policy objective is to minimize

$$
E_{t}\left(\pi_{t+2}-\pi *\right)^{2}=\left(E_{t} \pi_{t+2}-\pi *\right)^{2}+V_{t} \pi_{t+2}
$$

Although this assumption seems simplistic, Svensson (1997) has shown that the solution obtained in this manner is equivalent to the dynamic solution of a model in which the target is a weighted sum of all future squared deviations of inflation from the target level. Note also that equation (8) is analogous to equation (2) above in that it corresponds to an optimal forecast of inflation acceleration over the policy horizon, which is given by

$$
E_{t} \pi_{t+2}=\pi_{t}-a_{1}\left(1+b_{1}\right) \tilde{u}_{t}-a_{1} b_{2} r_{t}+\left(a_{3}\left(1+c_{3}\right)-a_{1} b_{3}\right) x_{t}
$$

The conditional variance of inflation is

$$
V_{t} \pi_{t+2}=\sigma_{\xi}^{2}
$$

Since the variance of inflation does not depend on the policy variable, the result is determined by certainty equivalence, that is, the optimal rule may be obtained by setting expected inflation equal to the target, $\pi^{*}$, and solving for the value of the policy variable. The optimal value of the policy variable is given by 


$$
\begin{gathered}
r_{t}^{*}=-\frac{1+b_{1}}{b_{2}} \tilde{u}_{t}+\frac{a_{3}\left(1+c_{3}\right)-a_{1} b_{3}}{a_{1} b_{2}} x_{t}+\frac{1}{a_{1} b_{2}}\left(\pi_{t}-\pi *\right) \\
=-\frac{1+b_{1}}{b_{2}}\left(\tilde{u}_{t}-n_{t}\right)+\frac{1}{a_{1} b_{2}}\left(\pi_{t}-\pi *\right)
\end{gathered}
$$

where the short-run NAIRU (defined as a deviation from $\bar{u}$ ) is:

$$
n_{t}=\frac{a_{3}\left(1+c_{3}\right)-a_{1} b_{3}}{a_{1}\left(1+b_{1}\right)} x_{t}
$$

Equation (9) is a variant of the Taylor (1993) rule, but which differs in that it is expressed in terms of unemployment rather than output. In addition, it allows for the reference point for monetary tightening in terms of the level of unemployment to be a short-run NAIRU rather than a fixed natural rate. In effect what this variation on the Taylor rules does is that it brings in additional information that helps forecast inflation in deriving an optimal setting of the policy instruments.

Even in this relatively simple setting, the short-run NAIRU $n_{t}$ is not a constant, but is instead a function of the exogenous variable $x$. If lags of inflation, unemployment and the policy variable appear in equations (5) and (6), their role in the policy rule -- and therefore in the definition of short-run NAIRU -- would be like that of $x$ in the model. Of course, if the only variable that helps predict inflation over the policy horizon, other than the unemployment rate, is a constant, then the NAIRU will be constant as in a more standard formulation. Note also that, like $\tilde{u}$, the short-run NAIRU of our theoretical model is measured in relation to $\bar{u}$. In empirical 
applications, we would want to focus on the equivalent of $n_{t}+\bar{u}$ as a measure of short-run NAIRU.

Equation (9) also helps to clarify the proper use of the NAIRU for policy purposes. The policy objective is not to drive unemployment to the NAIRU, which is a temporary and variable reference point, but to use the NAIRU unemployment gap as one indicator of the direction to move the policy variable, by an amount dictated by the coefficients of the model. Also, the NAIRU gap indicator is not to be interpreted in isolation, but must be weighed against the effect on the optimal setting of the policy variable suggested by the other indicator which is also included in the reaction function, the gap between actual and target inflation.

It is also important to recognize that our equation (9) variant of the Taylor rule is completely consistent with the result of Svensson (1997). Setting the policy instrument according to (9) is equivalent to setting expected inflation over the policy horizon equal to the inflation target $\pi^{*}$, which is the Svensson (1997) optimality condition if only inflation is in the objective function.

We can also draw some conclusions about the sign of the coefficient of $x$ in the definition of NAIRU, based on whether $x$ represents a supply or a demand effect. For example, if $x$ is a supply effect such as an oil price shock, then $a_{3}$ and $b_{3}$ would have the same sign. Since the other parameters in equation (10) were chosen to have positive values, the two terms in the coefficient would be offsetting and the net effect of $x$ on short-run NAIRU would be indeterminate. In contrast, if $x$ represents a demand effect, then $a_{3}$ and $b_{3}$ would have opposite signs and the two terms would be reinforcing. The sign of the effect is positive if the demand variable $x$ increases inflation and vice versa. In other words, a demand shock that raises inflation 
would lead to a higher value of short-run NAIRU, which implies more tightening given the same value of unemployment.

Supply and demand shocks also have differential effects on the overall implication about the optimal setting of the policy variable. The cumulation of supply effects would tend to drive both unemployment and inflation in the same direction, producing offsetting effects in equation (9). Cumulated demand effects, however, would drive inflation and unemployment in different directions, providing an unambiguous policy reaction. Therefore, demand effects that raise inflation should provoke a policy tightening.

\subsection{Output As Well As Inflation in the Objective Function}

Even when inflation is the only concern of policymakers, as in section 3.1 , the optimal policy assigns a significant role to the level of unemployment or to the unemployment gap, as seen in equation (9). In this section, we explore how policy should be conducted when policymakers include both inflation and output in their objectives. We do this by including a second term in the objective function, which now becomes

$$
E_{t}\left(\pi_{t+2}-\pi^{*}\right)^{2}+\lambda E_{t} \tilde{u}_{t+1}^{2}
$$

The economic significance of this change is that the policy objective assigns some weight to reducing the variability of unemployment around zero, which is the equilibrium level. ${ }^{6}$

The optimal value of the policy variable in this case is 


$$
r_{t}^{(\lambda)}=\frac{1}{\left(a_{1}^{2}+\lambda\right) b_{2}}\left(-\left(\left(1+b_{1}\right)\left(a_{1}^{2}+\lambda\right)-\lambda\right) \tilde{u}_{t}+\left(a_{1} a_{3}\left(1+c_{3}\right)-\left(a_{1}^{2}+\lambda\right) b_{3}\right) x_{t}+a_{1}(\pi t-\pi *)\right)
$$

The modification of the objective function to reflect an unemployment target changes the weights on $u, x$ and $\pi_{t}-\pi^{*}$ in the optimal policy rule, but does not affect its general form.

Specifically, the weight on $\tilde{u}_{t}$ relative to the weight on $\pi_{t}-\pi^{*}$ rises with $\lambda$. In the extreme, if the weight on unemployment becomes infinitely large ( $\lambda$ approaches infinity), the optimal rule simplifies to

$$
r_{t}^{(\infty)}=-\frac{b_{1}}{b_{2}} \tilde{u}_{t}-\frac{b_{3}}{b_{2}} x_{t}
$$

in which the inflation gap has disappeared and only an unemployment gap remains. This result may also be obtained by certainty equivalence, setting expected unemployment equal to its equilibrium level and solving for the value of the policy variable.

\section{The NAIRU and Policy-Making: Implications of Parameter Uncertainty}

4.1 Objective Function with Inflation Only

4.1.1 Uncertainty about the natural rate of unemployment

We begin to examine the consequences of uncertainty in the model of section 3 by looking at the effects of uncertainty regarding the natural rate of unemployment or, equivalently, the long-run NAIRU. We start with this particular question for two reasons. First, it seems that 
in the policy discussion on the use of the NAIRU, it is this question that is most frequently in the minds of the policymaker, although it is not always precisely formulated. Second, the examination of this narrower issue provides helpful intuition for the more general results that follow in the rest of this section.

Thus, consider a more focused version of the model of section 3 in which the traditional long-run NAIRU is the appropriate reference point for monetary policy in terms of the unemployment rate.

$$
\begin{aligned}
& \pi_{t}=\pi_{t-1}-a_{1}\left(u_{t-1}-\bar{u}\right)+\epsilon_{t} \\
& =\pi_{t-1}-a_{1} u_{t-1}+a_{0}+\epsilon_{t} \\
& u_{t}-\bar{u}=b_{1}\left(u_{t-1}-\bar{u}\right)+b_{2} r_{t-1}+\eta_{t}
\end{aligned}
$$

where $a_{0}=a_{1} \bar{u}$ and, as in section $3, \bar{u}$ is the natural rate and $r_{t}$ is the monetary policy variable. We write these equations explicitly in terms of $\bar{u}$ in order to focus on uncertainty with regard to this parameter. For the same reason, we assume that the parameters $b_{1}$ and $b_{2}$ in equation (6a) are known.

The second expression for equation (5a), under the natural stochastic assumptions, may be estimated using least squares. It is straightforward then to calculate the asymptotic distribution of the parameter estimates, which are consistent. In particular, we can derive that $T V\left(\hat{a}_{1}, \hat{a}_{0}\right)$, the asymptotic variance of the vector of estimates $\left(\hat{a}_{1}, \hat{a}_{0}\right)$ multiplied by the number of observations $T$, is 


$$
\frac{\sigma_{\epsilon}^{2}}{\sigma_{u}^{2}}\left[\begin{array}{cc}
1 & \bar{u} \\
\bar{u} & \bar{u}^{2}+\sigma_{u}^{2}
\end{array}\right]
$$

where $\bar{u}$ and $\sigma_{u}^{2}$ are the unconditional asymptotic mean and variance of $u_{t}$ and $\sigma_{\epsilon}^{2}$ is the variance of $\epsilon_{t}$. Now, if $J$ is the Jacobian of the transformation $\left(a_{1}, a_{0}\right) \mid \rightarrow\left(a_{1}, \bar{u}\right)=\left(a_{1}, a_{0} / a_{1}\right)$, then asymptotically, $T V\left(\hat{a}_{1}, \hat{\bar{u}}\right)=T J V\left(\hat{a}_{1}, \hat{a}_{0}\right) J^{\prime}$, which equals

$$
\left[\begin{array}{cc}
\sigma_{\epsilon}^{2} / \sigma_{u}^{2} & 0 \\
0 & \sigma_{\epsilon}^{2} / a_{1}^{2}
\end{array}\right]
$$

where we have made use of the fact that the unconditional mean of equation (5a) is $\overline{\Delta \pi}=0$.

The foregoing derivations may now be incorporated into the optimization problem of section 3 , again with the objective function $E_{t}\left(\pi_{t+2}-\pi *\right)^{2}$, but now

$$
E_{t} \pi_{t+2}=\pi_{t}-a_{1}\left(1+b_{1}\right)\left(u_{t}-\bar{u}\right)-a_{1} b_{2} r_{t}
$$

and

$$
V_{t} \pi_{t+2}=\sigma_{a_{1}}^{2}\left(\left(1+b_{1}\right)\left(-u_{t}+\bar{u}\right)-b_{2} r_{t}\right)^{2}+a_{1}^{2}\left(1+b_{1}\right)^{2} \sigma_{\bar{u}}^{2}+\sigma_{a_{1}}^{2} \sigma_{\bar{u}}^{2}\left(1+b_{1}\right)^{2}+\sigma_{\xi}^{2}
$$

In the expression for the variance, the terms that include $\sigma_{\bar{u}}^{2}$ do not depend on the policy variable.

Since the estimators of $\bar{u}$ and $a_{1}$ are orthogonal, the optimal rule will not depend on the uncertainty with regard to $\bar{u}$, as shown in the expression 


$$
r_{t}^{*}=-\frac{1+b_{1}}{b_{2}}\left(u_{t}-\bar{u}\right)+\frac{1}{1+\tau_{1}^{-2}} \cdot \frac{\pi_{t}-\pi^{*}}{a_{1} b_{2}}
$$

where $\tau_{1}=a_{1} / \sigma_{a_{1}}$.

Thus, uncertainty about the natural rate, in and of itself, does not affect the solution to the policymaker's optimization problem, as defined in this section and in section 3 . However, the uncertainty about the natural rate does increase the cost function because, as seen above, it increases the conditional variance of $\pi_{t+2}$. The uncertainty about the parameter $a_{1}$, the effect on inflation acceleration of the gap between unemployment and the natural rate, does figure in the optimal policy through the term $\left(1+\tau_{1}^{-2}\right)^{-1}$ which is a essentially a function of the $t$-statistic on $a_{1}$. Its effect, however, is not on the term containing the unemployment gap, but rather on the term containing the gap between current and target inflation. The greater the uncertainty about $a_{1}$, the lower is $\tau_{1}$ and therefore $\left(1+\tau_{1}^{-2}\right)^{-1}$, so the less weight the policymaker should place on the current inflation gap. This result is very robust, as it obtains in the models of subsequent sections, in which we introduce more complex specifications with fairly general parameter uncertainty.

\subsubsection{General Parameter Uncertainty}

Consider again the model defined by equations (5)-(7) of section 3.1, but assume now that there is uncertainty at time $t$ about the all the coefficients of the model $\left(a_{1}, a_{3}, b_{1}, b_{2}, b_{3}, c_{3}\right)$ and about the disturbance of the reduced form $(\xi)$, but that the 
uncertainty in all of these variables is pairwise orthogonal. Although these uncertainty assumptions are not entirely general -- on account of the assumed orthogonality -- they are more extensive than those that the previous literature has examined. ${ }^{7}$ The orthogonality assumptions are easily relaxed for coefficients belonging to the same equation, but the inclusion of the corresponding covariances does not provide greater intuition and is therefore not pursued here.

Thus at time $t$, the expectation and variance of inflation at time $t+2$ are given by

$$
E_{t} \pi_{t+2}=\pi_{t}-a_{1}\left(1+b_{1}\right) \tilde{u}_{t}-a_{1} b_{2} r_{t}+\left(a_{3}\left(1+c_{3}\right)-a_{1} b_{3}\right) x_{t}
$$

and

$$
\begin{aligned}
V_{t} \pi_{t+2}= & \left(a_{1}^{2} \sigma_{b_{1}}^{2}+\sigma_{a_{1}}^{2}\left(1+b_{1}\right)^{2}+\sigma_{a_{1}}^{2} \sigma_{b_{1}}^{2}\right) \tilde{u}_{t}^{2}+\left(a_{1}^{2} \sigma_{b_{2}}^{2}+\sigma_{a_{1}}^{2} b_{2}^{2}+\sigma_{a_{1}}^{2} \sigma_{b_{2}}^{2}\right) r_{t}^{2} \\
+ & \left(a_{3}^{2} \sigma_{c_{3}}^{2}+\sigma_{a_{3}}^{2}\left(1+c_{3}\right)^{2}+\sigma_{a_{3}}^{2} \sigma_{c_{3}}^{2}+a_{1}^{2} \sigma_{b_{3}}^{2}+\sigma_{a_{1}}^{2} b_{3}^{2}+\sigma_{a_{1}}^{2} \sigma_{b_{3}}^{2}\right) x_{t} \\
& +2 \sigma_{a_{1}}^{2}\left(\left(1+b_{1}\right) b_{2} \tilde{u}_{t} r_{t}+b_{2} b_{3} r_{t} x_{t}+\left(1+b_{1}\right) b_{3}\right) \tilde{u}_{t} x_{t}+\sigma_{\xi}^{2}
\end{aligned}
$$

where the values of the coefficients denote their expected values. ${ }^{8}$

As in section 3.1, the policy objective is to choose $r_{t}$ so as to minimize the objective function

$$
E_{t}\left(\pi_{t+2}-\pi *\right)^{2}=\left(E_{t} \pi_{t+2}-\pi *\right)^{2}+V_{t} \pi_{t+2}
$$

In this case, the optimal value of the policy variable is given by

$$
r_{t}^{*}=\frac{1}{1+\tau_{2}^{-2}}\left(-\frac{1+b_{1}}{b_{2}} \tilde{u}_{t}-\frac{b_{3}}{b_{2}} x_{t}+\frac{1}{1+\tau_{1}^{-2}}\left(\frac{\pi_{t}-\pi *}{a_{1} b_{2}}+\frac{a_{3}\left(1+c_{3}\right)}{a_{1} b_{2}} x_{t}\right)\right)
$$


where $\tau_{1}=a_{1} / \sigma_{a_{1}}$ and $\tau_{2}=b_{2} / \sigma_{b_{2}}$. Equation (11) can be rewritten as

$$
r_{t}^{*}=\frac{1}{1+\tau_{2}^{-2}}\left(-\frac{1+b_{1}}{b_{2}}\left(\tilde{u}_{t}-\left(n_{t}+\phi_{t}\right)\right)+\frac{1}{1+\tau_{1}^{-2}} \cdot \frac{1}{a_{1} b_{2}} \cdot\left(\pi_{t}-\pi *\right)\right)
$$

where

$$
\phi_{t}=-\frac{1}{1+\tau_{1}^{2}} \cdot \frac{a_{3}\left(1+c_{3}\right)}{a_{1}\left(1+b_{1}\right)} x_{t}
$$

Comparison of equations (9) and (12) indicates that the presence of uncertainty introduces two multiplicative terms of the form $\left(1+\tau_{i}^{-2}\right)^{-1}$. These terms are essentially functions of the $t$ statistics corresponding to the parameters $a_{1}$ and $b_{2}$, respectively, which correspond to the one-period-ahead effects of unemployment on inflation and of the policy variable on unemployment. All other variance-related terms in the objective function drop out of the calculation. When there is no uncertainty about $a_{1}$ and $b_{2}$, the two multiplicative terms become 1 , reverting to the certainty equivalent case of section 3.1 .

One of the two uncertainty effects, the one related to $b_{2}$, the coefficient on the policy variable in equation (6), takes a form that is predictable from the analysis by Brainard (1967). Specifically, as $\sigma_{b_{2}}$ rises, the term $\left(1+\tau_{2}^{-2}\right)^{-1}$ falls so that uncertainty about the magnitude of the effect of the policy variable leads to a partial policy reaction -- a reaction that is less than that in the certainty equivalence case.

In contrast, uncertainty about $a_{1}$, the effect of unemployment on the change in inflation in equation (5), has an effect not on the scale of the policy reaction, but rather on the weight 
applied to $\pi_{t}-\pi^{*}$ and on the reference point in terms of unemployment at which that reaction occurs. Specifically, as $\sigma_{a_{1}}$ rises, the term $\left(1+\tau_{1}^{-2}\right)^{-1}$ falls so that the weight on $\pi_{t}-\pi^{*}$ falls. A rise in $\sigma_{a_{1}}$ causes the term $\left(1+\tau_{1}^{2}\right)^{-1}$ and the absolute value of the adjustment term $\phi_{t}$ to rise. If $x$ has a positive impact on inflation (i.e., $a_{3} x_{t}$ is positive), then $\phi_{t}$ is negative and so the reference point for monetary tightening in terms of unemployment, $n_{t}+\phi_{t}$, falls.

The effect of uncertainty about $a_{1}$ on how the reference point responds to change in $x$ is somewhat more complex. The net effect on the reference point $n_{t}+\phi_{t}$ depends on whether $x$ is a supply or demand variable, as discussed in section 3.1. Consider the combined expression

$$
n_{t}+\phi_{t}=\left(\frac{1}{1+\tau_{1}^{-2}} \cdot \frac{a_{3}\left(1+c_{3}\right)}{a_{1}\left(1+b_{1}\right)}-\frac{b_{3}}{1+b_{1}}\right) x_{t}
$$

If $x$ is a supply variable, the direction of the effect of uncertainty on the magnitude of the reference point is unclear. It is clear, however, that as uncertainty about $a_{1}$ approaches infinity, the sign of the coefficient is the same as the sign of $-b_{3}$. If $x$ is a demand variable, uncertainty reduces the absolute magnitude of the reference point unambiguously.

\subsection{Output As Well As Inflation in the Objective Function}

We now modify the results of the previous subsection by assuming that the policy objective function includes both inflation and unemployment. As in section 3.2, the objective function becomes

$$
E_{t}\left(\pi_{t+2}-\pi^{*}\right)^{2}+\lambda E_{t} \tilde{u}_{t+1}^{2}
$$


The optimal value under parameter uncertainty is

$$
r_{t}^{(\lambda)}=\frac{1}{1+\tau_{2}^{-2}}\left(\left(-\frac{1+b_{1}}{b_{2}}+\frac{\lambda}{\left(a_{1}^{2}+\sigma_{a_{1}}^{2}+\lambda\right)}\right) \tilde{u}_{t}+\left(-\frac{b_{3}}{b_{2}}+\frac{a_{1} a_{3}\left(1+c_{3}\right)}{\left(a_{1}^{2}+\sigma_{a_{1}}^{2}+\lambda\right)}\right) x_{t}+\frac{a_{1}\left(\pi_{t}-\pi *\right)}{\left(a_{1}^{2}+\sigma_{a_{1}}^{2}+\lambda\right)}\right)
$$

The effect of including a target for unemployment, as represented by $\lambda$, is analogous to the effect of uncertainty about $a_{1}$. In the above equation, these two terms occur additively in the same expression in the terms corresponding to the exogenous variable and the inflation gap.

Only in the unemployment term does $\lambda$ appear separately. Intuitively, the reason for this is that uncertainty about $a_{1}$ makes the relationship expressed in equation (5) less reliable, so policy becomes more concerned with affecting the "intermediate target" of equilibrium unemployment.

If the weight on unemployment becomes infinitely large, the optimal rule simplifies to

$$
r_{t}^{(\infty)}=\frac{1}{1+\tau_{2}^{-2}}\left(-\frac{b_{1}}{b_{2}} \tilde{u}_{t}-\frac{b_{3}}{b_{2}} x_{t}\right)
$$

in which, as in the certainty equivalence case, the inflation gap has disappeared and only an unemployment gap remains. Here, the only effect of uncertainty is of the rescaling type, as identified by Brainard (1967).

\section{The NAIRU and Policy-Making: the Implications of Model Selection}


In this section, we discuss another type of uncertainty that affects the definition of shortrun NAIRU, its computation, and the policy rule that results from inflation targeting.

Specifically, we focus on uncertainty regarding the correct form of the basic model and the associated problem of model selection. Whereas in section 4 we assumed that the form of the model was known, but that the parameters were estimated with uncertainty, we now suppose that the policymaker ignores some key information variable in the optimization problem. ${ }^{9}$

In general, if inflation two periods ahead is the policy target, and if a variable helps predict inflation at that horizon, it is inefficient not to include the information in the model. For example, the models of sections 3 and 4 define the policy rule in terms of a short-run NAIRU, which in turn is a function of the exogenous variable $x$. What is the result of ignoring the predictive content of $x$ ? Alternatively, what is the cost of relying on a long-run equilibrium NAIRU (zero in this case) when a short-run informative NAIRU is available?

Thus, suppose that the policymaker ignores the presence of $x$ in the basic model (5)-(7). The values of $a_{3}$ and $b_{3}$ are implicitly set to zero, while the third equation is dropped altogether. Under these conditions, the constrained optimal rule for inflation targeting becomes

$$
\tilde{r}_{t}^{*}=\frac{1}{1+\tau_{2}^{-2}}\left(-\frac{1+b_{1}}{b_{2}} \tilde{u}_{t}+\frac{1}{1+\tau_{1}^{-2}}\left(\frac{\pi_{t}-\pi *}{a_{1} b_{2}}\right)\right)
$$

We know, of course, that the value of the objective function has to be higher (i.e., worse) when evaluated at this constrained optimum that when evaluated at the unconstrained optimum $r_{t}^{*}$ as in section 4.1. In fact, we can calculate the difference between the constrained and unconstrained values as 


$$
\frac{1}{1+\tau_{2}^{-2}} \cdot \frac{1}{a_{1}^{2}+\sigma_{a_{1}}^{2}} \cdot\left(a_{1} a_{3}\left(1+c_{3}\right)-\left(a_{1}^{2}+\sigma_{a_{1}}^{2}\right) b_{3}\right)^{2} x_{t}^{2}
$$

Somewhat surprisingly, uncertainty about $b_{2}$ ameliorates the left-out-variable problem. ${ }^{10}$ Uncertainty about $a_{1}$, in contrast, can make matters worse.

The left out variable problem can also increase uncertainty regarding the estimates of the included coefficients, with consequences for the size of the policy response or the reference point for monetary tightening in terms of unemployment. To see this, suppose the inflation equation (5) is estimated by ordinary least squares, leaving out the variable $x$, after rewriting it in the following form

$$
\pi_{t}-\pi_{t-1}=-a_{1} \tilde{u}_{t-1}+\epsilon_{t}
$$

One implication of leaving out $x$, well known from econometrics textbooks, is that the estimate of $a_{1}$ may be biased. This occurs unless $x$ and $u$ are contemporaneously uncorrelated. ${ }^{11}$ However, even if the two regressors are indeed uncorrelated so that the estimate of $a_{1}$ is unbiased, uncertainty in the estimate is greater by the amount

$$
\frac{\sum_{t} \pi_{t}^{2}}{\sum_{t} \tilde{u}_{t-1}^{2}} \cdot \frac{R_{u}^{2}-R_{c}^{2}}{n}
$$

where the numerator of the last term is the difference between the $R^{2}$ s of the unconstrained and the constrained models. Thus, excluding the variable $x$ from the model, in addition to producing a policy rule that improperly excludes $x$, increases uncertainty about $a_{1}$. One possible 
consequence is that, for the reasons provided in section 4 , the policymaker may react to the higher level of $\sigma_{a_{1}}$ by adjusting the weight on $\pi_{t}-\pi^{*}$ downward and by increasing the absolute size of the NAIRU adjustment $\phi_{t}$.

\section{Empirical Estimates of the short-run NAIRU}

\subsection{Empirical Evidence on the Importance of Uncertainty}

Although our theoretical framework shows qualitatively the effects of uncertainty on how monetary policy should be conducted, it cannot tell us whether these effects are economically important. To examine this question, we estimate in this section a simple NAIRU gap model for the United States to obtain measures of uncertainty and to assess how these measures affect our view of the optimal reaction of monetary policy to movements in unemployment relative to short-run NAIRU. In order to have in the model a simple lag structure that mimics that of the theoretical model of equations (5)-(7), we start by estimating a model with annual U.S. data over the period 1956 to 1996 . The model is

$$
\begin{aligned}
& \pi_{t}-\pi_{t-1}=\alpha_{0}+-\alpha_{1} u_{t-1}+\alpha_{2} u_{t-2}+\epsilon_{t} \\
& u_{t}=\beta_{0}+\beta_{1} u_{t-1}+\beta_{2} u_{t-2}+\beta_{3} r_{t-1}+\beta_{4} r_{t-2}+\eta_{t}
\end{aligned}
$$

where $\pi$ is the log change in the CPI from December of year $t-1$ to December of year $t, u$ is the unemployment rate in December of year $t$ and $r$ is the average monthly three-month Treasury bill 
during year $t$. Note that $\alpha_{1}, \beta_{3}$ correspond to $a_{1}, b_{2}$ in the theoretical model, and that the key uncertainty ratios $\tau_{1}^{-2}, \tau_{2}^{-2}$ will be based on the former. The results are presented in table 1 .

These estimates provide some guidelines regarding the importance of uncertainty for monetary policy in this context. First, the adjustments to the unemployment reference point and to the policy reaction as a result of parameter uncertainty are not large. The key parameters are estimated with some precision, and the implied multiplicative adjustment factors are both close to 1 . The Brainard-type adjustment -- a 2.5 percent reduction -- is particularly small, suggesting that the magnitude of the policy reaction should only be shaded down slightly to reflect parameter uncertainty. However, the unemployment effect adjustment is also less than 5 percent.

These results are confirmed by looking at the implicit optimal policy that corresponds to the two-year ahead inflation target of the theoretical model in which only inflation is included in the objective function. The rule that results is very similar to the simple Taylor (1993) rule when adjustments are made for the fact that Taylor's rule was defined in terms of quarterly data and an output gap. The annual and quarterly results are presented in table 2 . If $\delta$ is the weight on the lagged interest rate in the annual model, the corresponding quarterly lag is assigned a weight of $\delta^{1 / 4}$ and the weights on the inflation and unemployment lags are divided by $1+\delta^{1 / 4}+\delta^{2 / 4}+\delta^{3 / 4}$. A rule based on the output gap is obtained by applying a simple Okun's law adjustment, dividing the unemployment weight by 2 .

The table confirms that the practical significance of parameter uncertainty is quite small. Furthermore, the quarterly results with the output gap are remarkably similar, even numerically, to the parameters suggested by Taylor (1993). The only key difference is that the interest rate is 
assumed to be much more persistent here, since Taylor did not include a lagged interest rate in the form of his rule. ${ }^{12}$

\subsection{Empirical Estimates of the Short-Run NAIRU}

In this section, we present estimates of the short-run NAIRU. For these purposes, we return to the more general model of equations (2)-(4) and estimate the equations with monthly data from January 1954 to November 1997, using a 12-month-ahead, 12-month horizon $(j=k=12)$ and 12 lags of both the change in inflation and unemployment. ${ }^{13}$ Figure 1 shows the estimated short-run NAIRU together with the contemporaneous unemployment rate, as well as the shortrun NAIRU gap. This figure demonstrates the high variability of the short-run NAIRU, in contrast with long run measures designed to estimate a natural rate as in Gordon (1997) and Staiger, Stock and Watson (1997a,b). For example, consider a version of our equation (1), which may be used to estimate a constant $\bar{u}$ that is comparable to the long-run measure of those papers:

$$
\Delta \pi_{t}=\beta(L)\left(u_{t}-\bar{u}_{t}\right)+\gamma(L) \Delta \pi_{t-1}+\epsilon_{t}
$$

When estimated over the same period as equations (2)-(4), the estimate of $\bar{u}$ is 6.1 percent, as shown in figure 1.

Staiger, Stock and Watson (1997a) have pointed out that such estimates of a constant long-run NAIRU tend to be quite imprecise. Using the delta method in an equation similar to (1'), they obtain an estimate of $\bar{u}=6.2$ percent, with a standard error of about 0.6 . Our estimate of $\bar{u}=6.1$ has a standard error of 0.43 , which is somewhat smaller -- perhaps partly because of our 
larger sample -- but is of the same order of magnitude. Estimates of the short-run NAIRU $n_{t}$ are more precise. The standard error of $n_{t}$ is a time-varying function of the values of the variables in expression (4). Over the sample period, the standard errors range from 0.11 to 0.42 , with a mean of 0.20 , less than half of the standard error of $\bar{u} .^{14}$

Thus, the short-run NAIRU is estimated with more than twice the precision than the standard long-run NAIRU. The practical significance of this result, however, is limited, since we have shown in the theoretical sections that this type of uncertainty plays no role in the determination of the policy rule. Nevertheless, a reduction in the uncertainty may produce a reduction is the value of the cost function, as shown in section 5 , even if the policy rule remains unaltered.

6.3 A case study: recent signals from a short-run NAIRU

Using the estimates of the NAIRU gap from section 6.2, we now examine the hypothetical results of using the methodology of this paper in the of conduct monetary policy in the U.S. since June 1992, when the unemployment rate began a prolonged decline. The results will of course be somewhat simplistic, but they may provide some general support for the concepts developed in this paper.

If we refer to one of the policy rules in the theoretical part of the paper, say to equation (9), we note that the appropriate interest rate is determined essentially by two gaps: the difference between unemployment and short-run NAIRU and the difference between actual and target inflation. We present in figure 2a the gap between short-run NAIRU and unemployment (signed 
so that a positive value indicates that monetary policy should be tightened) and the level of inflation (12 previous months) since 1992.

From June 1992 to the end of 1993, declining unemployment brought the NAIRU gap from levels suggesting, if anything, the need for ease to relatively neutral levels. Meanwhile inflation declined over the period and, in fact, continued to decline into the beginning of 1994. Beginning in 1994, however, the NAIRU gap became positive and remained so until early 1995 , suggesting a need for tightening. In addition, inflation stopped declining, remaining around the 3 percent level. These two factors combined are consistent with the monetary tightening undertaken by the Federal Reserve throughout 1994 and into early 1995.

Since then, the NAIRU gap has indicated some pressure to tighten twice, in 1996 and 1997. In the first case, the pressure from the NAIRU gap was accompanied by a rise in inflation. Even though inflation subsided toward the end of the year, this episode may seem somewhat inconsistent with the absence of further tightening. Figure $2 \mathrm{~b}$ suggests one reason for this result. Figure $2 \mathrm{~b}$ presents the results of repeating the analysis of figure $2 \mathrm{a}$, but using core inflation (excluding food and energy prices) instead of total inflation. Core inflation tends to be a better signal of persistent changes in inflation than total inflation.

Figure $2 \mathrm{~b}$ shows both the level of core inflation as well as the gap between unemployment and the short-run NAIRU computed using core inflation in equations (2)-(4). Comparisons of the two panels of figure 2 suggests that the effect of using core inflation in the calculation of the NAIRU gap is very slight. But core inflation was falling in 1996, in contrast to the rising total inflation, and this fall may have offset the tightening signals from the NAIRU gap. 
In 1997 , the pressure arising from the unemployment gap seems stronger than in the previous year. Inflation, however, both total and core, moved downward again, offsetting at least partially the signals from the NAIRU gap indicator. Arguably, only during 1994 and early 1995 were there consistent signals for tightening and this is when the Federal Reserve engaged in most of its monetary tightening.

In order to evaluate the net effect of the unemployment and inflation indicators, it would be helpful to summarize the information in a single measure, as in the policy rules of table 2 . We would like to do this, not to explain actual policy, but to suggest how the theoretical constructs of this paper could be used in practice. However, this is a problem for two reasons. First, we would have to construct a full optimization model in the context of the monthly equations, which is beyond the scope of the present paper. ${ }^{15}$ Second, we would have to know or make an assumption about the target level of inflation. Thus, we present only a limited version of a policy rule in which we deal with those problems as follows.

First, we take the weights for the NAIRU and inflation gaps from the annual results of table 2 allowing for uncertainty, making allowance also for the monthly frequency of our data. Since the coefficient of the lagged interest rate, $\delta^{1 / 12}=0.98$, is very close to 1 , we further simplify by assuming that the weights are used to calculate a monthly change in the interest rate. We then divide the annual weights by $1+\delta^{1 / 12}+\ldots+\delta^{11 / 12}$ to obtain weights of -0.23 for the NAIRU gap and 0.15 for the inflation gap with total inflation, and -0.25 and 0.19 , respectively, using core inflation. ${ }^{16}$ To deal with the second problem, the fact that the inflation target is unknown, we scale the results so that the policy rule with total inflation is neutral, on average, over the period since June 1992. This assumption is equivalent to an inflation target of 3 percent. 
The results are presented as the solid line in the two panels of figure 3 . Note that the weighted results are consistent with our earlier discussion of the individual components. In panel $3 \mathrm{a}$, which contains the results using the total $\mathrm{CPI}$, the strongest signal for tightening comes during 1994. Note also, however, that there were distinct signals for tightening in 1992-93 and 1996-97, and that there were fairly strong signals for easing at the beginning and towards the end of the sample period. In panel $3 \mathrm{~b}$, which contains results using the core CPI, there are also strong signals to tighten in 1994, but because the core inflation rate was higher than total CPI in late 1992 and early 1993, there are also strong signals to tighten in this period. In contrast to panel 3a, the results with the core CPI do not suggest any need to tighten in 1996.

We may contrast these results with a rule based on the standard unemployment gap -- the gap between unemployment and a constant long-run NAIRU. The results are presented as the dashed line in the two panels of figure 3 . To obtain weights that are consistent with the assumption of a constant NAIRU, we estimated equations (12) and (13) without the second lag of unemployment, which produces an estimate of NAIRU that is constant. These new weights are -0.35 for the NAIRU gap and 0.34 for the inflation gap using total inflation, and -0.36 and 0.37 , respectively, using core inflation. Note, however, that if we use of the same weights as before, the qualitative results are the same as with these weights.

The results for the long-run NAIRU gap, which are driven by the large steady decline in unemployment over this period, are fairly robust. The main feature of the alternative rule is that it argues for easing throughout the first part of the period, and then for tightening throughout the second part of the period. What this rule misses is that a long-run natural rate is not the best reference point for unemployment if the goal is to target inflation in the short run. 


\section{Summary and Conclusions}

In this paper, we examine how a variant of the NAIRU concept can be usefully employed in the conduct of monetary policy. By thinking of NAIRU in this way, we obtain insights that might be quite useful to monetary policymakers. Because there are quite a few results sprinkled throughout the paper, we list the main ones here.

- The NAIRU concept that is useful for the conduct of monetary policy differs from the estimate of the natural rate of unemployment, the long-run concept used previously by many researchers. Instead, NAIRU can be viewed as a short-run construct, which is related to past levels of unemployment and inflation as well as other economic variables, that helps forecast future accelerations or decelerations of inflation.

- The short-run NAIRU should be viewed not as a target for policy, but as helping to define the reference point which policymakers can compare to the current rate of unemployment to derive a signal for the appropriate stance of policy. Furthermore, as long as inflation is an element in the policymakers' objective function, the NAIRU gap is not the only signal that should affect the setting of policy instruments: the deviation of inflation from its target level also has an important role in the determination of the appropriate stance of policy.

- The policy rule that comes out of our analysis is a variant of a Taylor rule using an unemployment gap rather than an output gap, but has one major difference from more 
standard formulations. The standard Taylor rule implicitly assumes that the reference point to which unemployment should be compared in the unemployment gap term is constant, while in our formulation, the reference point is related to the short-run NAIRU which can have substantial short-run fluctuations over time.

- Uncertainty about the level of the NAIRU has no influence on the setting of policy instruments, although it does affect the value of the objective function. This type of uncertainty makes the economy worse off, but does not alter policy behavior.

- Uncertainty about the effect of unemployment on inflation leads to an additive adjustment to the short-run NAIRU to calculate the reference point for monetary tightening in terms of the level of unemployment. In addition, uncertainty about the unemployment effect on inflation changes the weight on the inflation gap in the policy rule.

- Uncertainty about effect of the policy variable leads to a scaling down of the reaction of the policy variable, the well-known Brainard (1967) result.

- Uncertainty about model selection can have important effects on the form of the policy rule. In particular, if a constant NAIRU is used -- as occurs if NAIRU is viewed as a long-run concept -- so that information about the state of the economy that could be used to forecast inflation is ignored, the performance of the policy rule can be substantially 
worse. In addition, leaving out relevant variables that help forecast inflation increases the uncertainty about the effect of unemployment on inflation, with the resulting implications described above.

- Although parameter uncertainty has potentially large effects on how policy should be conducted, our empirical results suggest that parameter uncertainty may not be all that important for the setting of policy. We find some evidence of changes in the policy rule resulting from the parameter uncertainty we explored in our theoretical model, but these effects are very modest. They affect the weights in the policy rule by less than five percent in both the case of uncertainty about the impact of unemployment and the case of uncertainty about the effect of the policy variable.

- Estimates of the short-run NAIRU are highly variable over time. However, there is a fair degree of precision in these estimates.

- Substantial positive NAIRU gap estimates arose throughout 1994 and early 1995 and in parts of 1996 and 1997. However, core inflation was substantially lower in 1996 and 1997 than in 1994 . Thus the one period since June 1992 during which there were consistent signals for tightening occurred during 1994 and early 1995, which is when the Fed engaged in most of its monetary tightening. 
These results suggest that a short-run NAIRU is indeed a useful concept and that it can be used by policymakers, particularly in deciding on how monetary policy should be conducted. However, there are some subtle issues in how the short-run NAIRU concept might be used correctly. First, because our view of NAIRU sees it as a short-run construct, it is dangerous to think of NAIRU as a potential target for unemployment which stays around a particular value, such as 6 percent, for any period of time. Second, deviations of inflation from its target are every bit as important a factor in thinking about setting policy as is the NAIRU gap. Third, uncertainty about parameter values and model selection do have effects on the optimal setting of policy instruments, but do not appear to be a barrier to a useful role for the NAIRU concept in policy decisions.

We hope that this paper helps resurrect NAIRU as a useful concept, but only if it is used properly. As we have shown, a short-run NAIRU is a useful construct because it helps tell policymakers what might happen to inflation in the future. Furthermore, the model of this paper suggests that policymakers may want to avoid the impression that an objective of policy is to raise unemployment when it falls below NAIRU or to lower it when it is above NAIRU. To think of policy in this way might lead the public to think that policymakers are against low unemployment, an outcome that can reduce support for central bank efforts to control inflation. 


\section{Footnotes}

1. If price stability has already been achieved, then inflation falling below its target is every bit as damaging as a rise in inflation above the target. Thus, in this situation monetary policy must also be just as preemptive against declines in inflation below target levels.

2. See, for example, Stiglitz (1997), Gordon (1997), Staiger, Stock and Watson (1997a,b), Economic Report of the President (1997). For a history of NAIRU, see Espinosa-Vega and Russell (1997). The NAIRU acronym would better be expressed as NIIRU (the non-increasing inflation rate of unemployment) because it is the unemployment rate at which inflation is expected to neither increase or decrease.

3. See, for example, Stiglitz (1997), Gordon (1997), Staiger, Stock and Watson (1997a,b), Economic Report of the President (1997). For a history of NAIRU, see Espinosa-Vega and Russell (1997). The NAIRU acronym would better be expressed as NIIRU (the non-increasing inflation rate of unemployment) because it is the unemployment rate at which inflation is expected to neither increase or decrease.

4. The variables $x$ differ from $z$ in the Gordon (1997) and Staiger, Stock and Watson (1997a,b) equations in that $z$ represents primarily supply shocks that are contemporaneous with the dependent variable, whereas $x$ is more general in that it includes any predetermined variables other than unemployment and inflation (and their lags) that help predict future inflation. 5. Equation (4) is a generalization of the model of short-run NAIRU in Estrella (1997). After writing this paper, we discovered that Layard and Bean (1988) also have a similar definition of short-run NAIRU in the context of a one-period change in inflation. 
6. Once again, this is a relatively simple objective function designed to highlight the key points of this paper. A more complex dynamic solution of a similar model may be found in Svensson (1997), which exhibits properties that are qualitatively analogous to those of the simpler model of this paper.

7. Other papers that look at the effect of parameter uncertainty in a similar context are Svensson (1997), Clarida, Gali and Gertler (1997), and Wieland (1997).

8. This convention economizes on notation and is correct by definition if the coefficient estimates are unbiased.

9. The complementary problem of including too many variables in the model is in principle less serious, since consistent parameter estimates should assign zero weight to the superfluous variables.

10. The intuition is that as uncertainty about $b_{2}$ grows, the optimal response of the policy variable $r$ is reduced so that there is less loss from using the incorrect model.

11. See, for instance, Theil (1971), section 11.2. This problem may be bypassed formally by thinking of $x$ as the component of the additional variable that is uncorrelated with $u$.

12. Recent estimates of the Taylor rule by Rudebusch and Svensson (1998) and Rotemberg and Woodford (1998), among others, suggest that the persistence parameter is close to 1 . Fuhrer and Moore (1995) assume that it equals 1.

13. Somewhat surprisingly, extending the horizon to $j+k=60$ months or even longer does not materially affect the point estimates of the short-run NAIRU. Of course, the fit of the equation deteriorates with longer horizons. 
14. All our standard errors are estimated consistently using the Newey-West (1987) technique with a 24-lag window.

15. A model along those lines has been developed for the U.S. in Clarida, Gali and Gertler (Forthcoming). See also the references in that paper.

16. Adjusting fully for coefficient uncertainty would require, in addition to the adjusted weights, an adjustment to short-run NAIRU corresponding to the term $\phi_{t}$ defined in section 4.1.2. We do not make this adjustment here because our equation for monthly NAIRU is essentially a reduced form and the components are difficult to disentangle, and also because the coefficient of the adjustment factor $\left(1+\tau_{1}^{2}\right)^{-1} \leq 0.065$ is empirically small. 


\section{References}

Brainard, William. 1967. "Uncertainty and the Effectiveness of Policy." American Economic Review, 411-425.

Clarida, Richard, Jordi Gali and Mark Gertler. Forthcoming. "The Science of Monetary Policy." Journal of Economic Literature.

Council of Economic Advisers. 1997. Economic Report of the President, 45-54.

Espinosa-Vega, Marco A. and Steven Russel1. 1997. "History and Theory of the NAIRU: A Critical Review." Economic Review, Federal Reserve Bank of Atlanta, 4-25.

Estrella, Arturo. 1997. “Aggregate Supply and Demand Shocks: A Natural Rate Approach.” Federal Reserve Bank of New York Research Paper No. 9737.

Friedman, Milton. 1968. "The Role of Monetary Policy." American Economic Review, 58:121.

Fuhrer, Jeffrey C. and George R. Moore. 1995. "Monetary Policy Trade-Offs and the Correlation between Nominal Interest Rates and Real Output." American Economic Review, 219-239.

Gordon, Robert J. 1997. "The Time-Varying NAIRU and its Implications for Economic Policy." Journal of Economic Perspectives, 11:11-32.

Layard, Richard and Charles R. 1988. "Why Does Unemployment Persist?” Centre for Labour Economics, London School of Economics, Discussion Paper No. 321 (August).

Modigliani, Franco and Lucas Papademos. 1975. "Targets for Monetary Policy in the Coming Year." Brookings Papers on Economic Activity, 1:141-63. 
Newey, Whitney K. and Kenneth D. West. 1987. “A Simple-Positive Semi-Definite,

Heteroskedasticity and Autocorrelation Consistent Covariance Matrix," Econometrica, 55:703-708.

Phelps, Edmund. 1968. “Money-Wage Dynamics and Labor-Market Equilibrium.” Journal of Political Economy, 76:678-711.

Rotemberg, Julio J. and Michael Woodford. 1998. "Interest Rate Rules in an Estimated StickPrice Model." This conference.

Rudebusch, Glenn D. and Lars E.O. Svensson. 1998. "Policy Rules for Inflation Targeting." This conference.

Staiger, Douglas, James H. Stock and Mark W. Watson. 1997a. "How Precise are Estimates of the Natural Rate of Unemployment?" In Reducing Inflation: Motivation and Strategy, Christina D. Romer and David H. Romer, eds., Chicago: University of Chicago Press.

Staiger, Douglas, James H. Stock and Mark W. Watson. 1997b. "The NAIRU, Unemployment and Monetary Policy." Journal of Economic Perspectives, 11:33-49.

Stiglitz, Joseph. 1997. "Reflections on the Natural Rate Hypothesis." Journal of Economic Perspectives, 11:3-10.

Svensson, Lars E.O.. 1997. “Inflation Forecast Targeting: Implementing and Monitoring Inflation Targets." European Economic Review, 41:1111-1146.

Taylor, John B. 1993. "Discretion Versus Policy in Practice." Carnegie-Rochester Conference Series on Public Policy, 39:195-214.

Theil, Henri. 1971. Principles of Econometrics. New York: Wiley. 
Wieland, Volker. 1997. "Monetary Policy and Uncertainty about the Natural Unemployment Rate." Manuscript, Board of Governors of the Federal Reserve System. 
Table 1. Estimates of annual U.S. model (1956 to 1996)

\begin{tabular}{|c|c|c|c|}
\hline Coefficient & Estimate & $t$ & $\left(1+t^{-2}\right)^{-1}$ \\
\hline \hline$\alpha_{0}$ & 1.67 & 1.49 & \\
\hline$\alpha_{1}$ & 1.24 & 4.68 & .956 \\
\hline$\alpha_{2}$ & .98 & 3.70 & \\
\hline$R^{2}$ & .366 & & \\
\hline$\beta_{0}$ & .70 & 1.31 & \\
\hline$\beta_{1}$ & 1.00 & 7.06 & \\
\hline$\beta_{2}$ & -.23 & -2.18 & \\
\hline$\beta_{3}$ & .48 & 6.30 & \\
\hline$\beta_{4}$ & -.36 & -3.84 & \\
\hline$R^{2}$ & .833 & & \\
\hline
\end{tabular}


Table 2. Implicit interest rate rules

\begin{tabular}{|l|l|c|c|}
\hline Rule & \multicolumn{2}{|l|}{$\begin{array}{l}\text { Weight on } \\
\text { Lagged interest rate }\end{array}$} & $\begin{array}{l}\text { Inflation gap } \\
\text { or output gap }\end{array}$ \\
\hline Unadjusted: & & & \\
Annual & .77 & 1.70 & -2.56 \\
\hline Quarterly & .94 & .47 & -.70 \\
\hline With output gap & .94 & .47 & .35 \\
\hline Uncertainty adjusted: & & & \\
Annual & .77 & 1.59 & -2.49 \\
\hline Quarterly & .94 & .44 & -.69 \\
\hline With output gap & .94 & .44 & .34 \\
\hline
\end{tabular}


Figure 1. Short-run NAIRU, unemployment and the short-run NAIRU gap Jan. 1954 to Nov. 1997

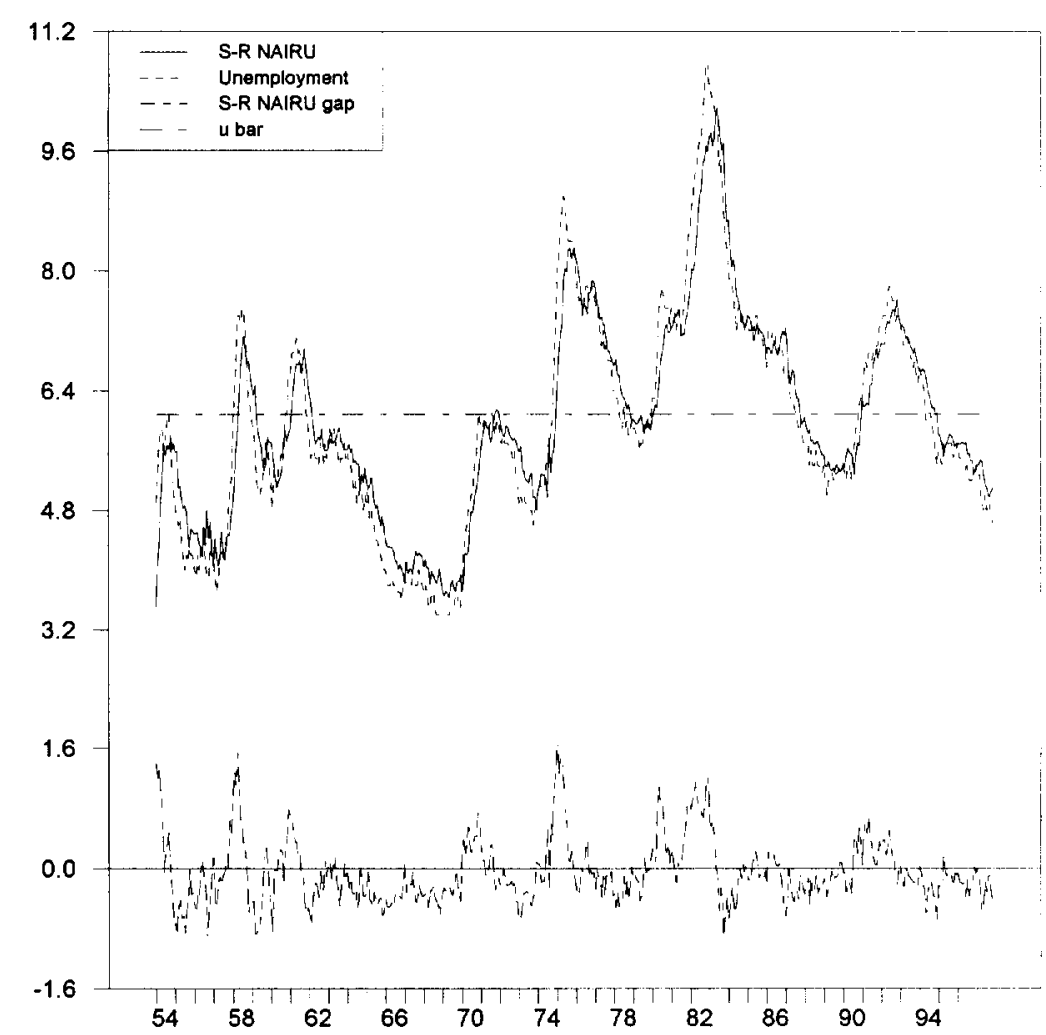


Figure 2. NAIRU gap and inflation, June 1992 to Nov. 1997
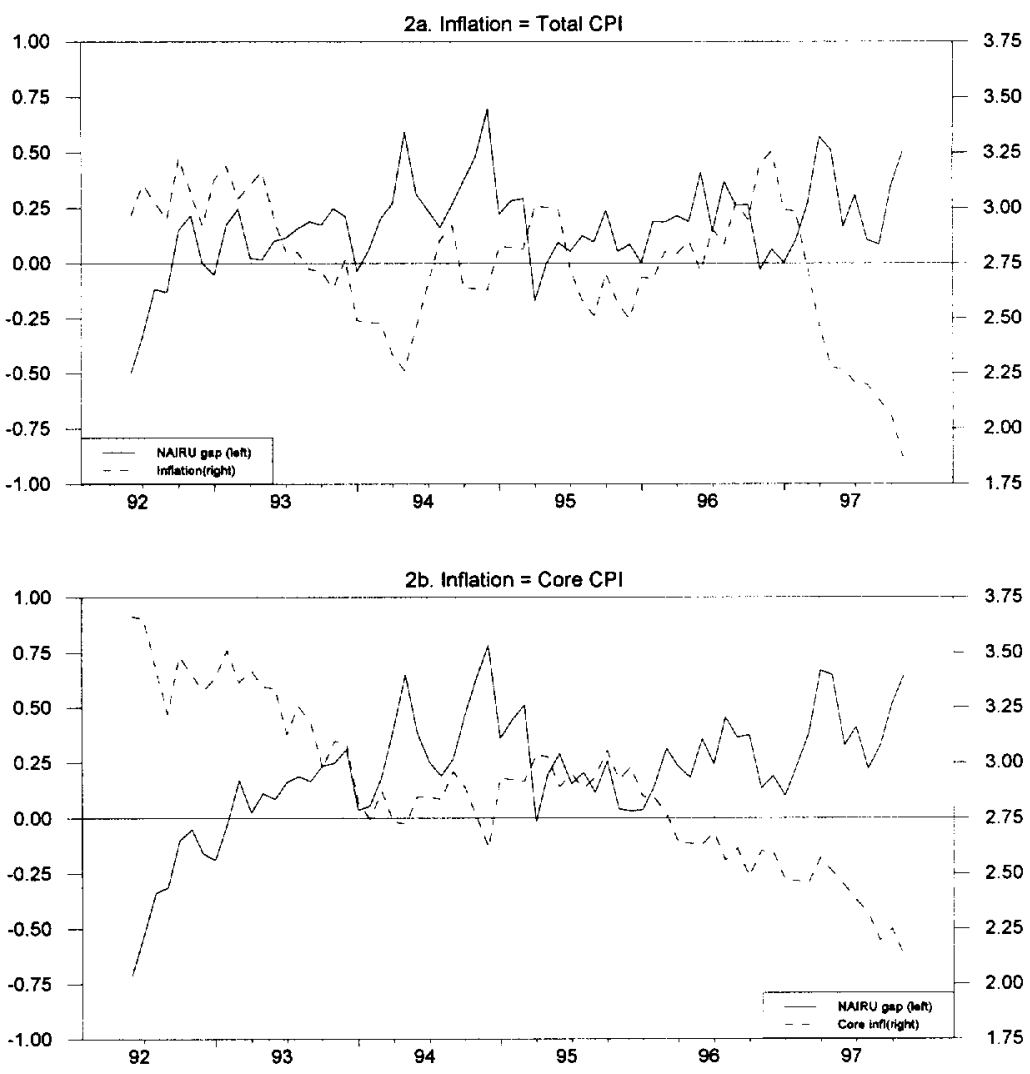
Figure 3. Simple policy rules based on short-run NAIRU (solid line) and long-run NAIRU (dashes), June 1992 to Nov. 1997
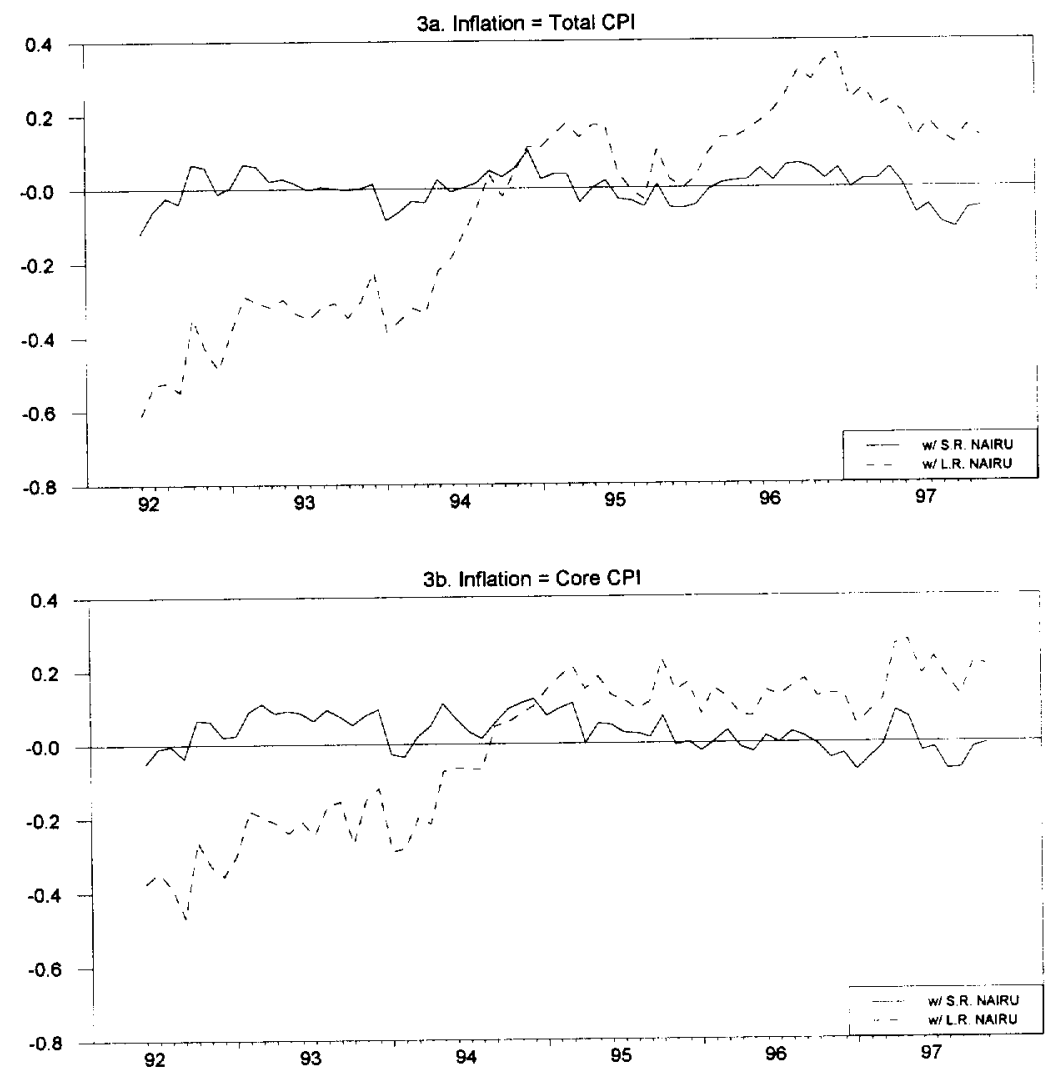